\title{
Uso de la escala de evaluación radiológica de edema pul- monar (Rale) en pacientes con Covid 19
}

\section{Use of the radiological assessment scale of pulmonary edema (Rale) In patients with Covid 19}

Recibido: 25/06/2021 Aceptado: 29/07/2021
Roberto Carlos Barrientos Ramirez Universidad de San Carlos de Guatemala, rocabarrientos@gmail.com https://orcid.org/0000-0001-9242-2494

\section{Referencia del artículo}

Barrientos Ramirez, R. C. (2021). Uso de la escala de evaluación radiológica de edema pulmonar (Rale) en pacientes con Covid 19. Revista Diversidad Científica, 1(1). 53-60. DOI: https://doi.org/10.36314/diversidad.v1i1.7

\section{Resumen}

INTRODUCCIÓN: el edema pulmonar se define como la extravasación anormal de líquido en el compartimento extravascular del pulmón, estos eventos obstaculizan la adecuada oxigenación sanguínea y por ende conllevan a la hipoxia tisular. Los hallazgos más frecuentes que se han asociado al Covid-19 son las opacidades del espacio aéreo en forma de consolidaciones y/u opacidades en vidrio deslustrado, con distribución típicamente bilateral, periférica y de predominio en los campos inferiores. OBJETIVO: describir la Score Rale como uso diagnóstico de edema pulmonar en pacientes con Covid 19. MÉTODOS: para el desarrollo de la monografía se realizó una revisión bibliográfica basada en fuentes primarias y secundarias. CONCLUSIÓN: la Score Rale se puede utilizar para evaluar tanto la extensión del edema pulmonar como la gravedad del Síndrome de dificultad respiratoria aguda -SDRA-, de forma rutinaria, segura y económica en todos los pacientes con SDRA. Esta escala permite evaluar la gravedad y extensión de la afectación pulmonar del Covid-19 y controlar la respuesta al tratamiento.

Palabras clave: Covid-19, Edema pulmonar, Score Rale, evaluación radiológica 


\begin{abstract}
INTRODUCTION: pulmonary edema is defined as the abnormal extravasation of fluid in the extravascular compartment of the lung, these events impede adequate blood oxygenation and therefore lead to tissue hypoxia. The most frequent findings that have been associated with Covid-19 are airspace opacities in the form of consolidations and / or ground-glass opacities, with a typically bilateral, peripheral distribution and predominantly in the lower fields. OBJECTIVE: to describe the Rale Score as a diagnostic use of pulmonary edema in patients with Covid 19. METHODS: for the development of the monograph, a bibliographic review based on primary and secondary sources was carried out. CONCLUSION: the Rale Score can be used to assess both the extent of pulmonary edema and the severity of Acute Respiratory Distress Syndrome-ARDS, in a routine, safe and economical way in all patients with ARDS. This scale makes it possible to assess the severity and extent of the lung involvement of Covid-19 and monitor the response to treatment.
\end{abstract}

Keywords: Covid-19, Pulmonary edema, Score Rale, radiological evaluation 


\section{Introducción}

El edema pulmonar se define como la extravasación anormal de líquido en el compartimento extravascular del pulmón, estos eventos obstaculizan la adecuada oxigenación sanguínea y por ende conllevan a la hipoxia tisular. En el edema agudo de pulmón se pueden encontrar múltiples signos e imágenes que nos van a orientar al diagnóstico radiológico temprano de esta patología, a este respecto, la radiografía de tórax continúa siendo un método no invasivo, rápido y de bajo costo.

Es de suma importancia reconocer los cambios radiográficos producidos durante la instauración del edema agudo de pulmón, el propósito de realizar un diagnóstico asertivo y temprano contribuye con una disminución en la morbilidad y mortalidad por esta causa. Una adecuada técnica en el momento de la toma de las radiográficas de tórax, proporcionará una valiosa herramienta, principalmente si se logra obtener la placa de rayos $\mathrm{X}$ en inspiración y con el paciente en posición erguida.

El objetivo de esta investigación es describir la Score Rale como método diagnóstico en pacientes con Covid-19 y edema pulmonar. Esta escala es una modificación de Score Rale, diseñado para estimar el grado de edema pulmonar en el SDRA.

\section{Contenido \\ Covid-19 y la evaluación radiológica del edema pulmonar}

El origen de los coronavirus de importancia médica, incluidos los coronavirus humanos, parece ser zoonótico. En particular, los betacoronavirus zoonóticos están filogenéticamente relacionados con coronavirus de murciélagos, los cuales podrían haber sido su fuente para el hombre, ya sea directamente o a través de un hospedero intermediario; dicho intermediario para el SARSCoV fue la civeta, un animal silvestre del grupo de los vivérridos, y para el MERS-CoV fue el dromedario. Aún no es claro cuál pudo haber sido el intermediario para el SARS-CoV-2, o si pasó directamente del murciélago al humano (AVMA, 2020).

De acuerdo con la OPS/OMS (2021) las definiciones de los casos se establecen en caso sospechoso, caso probable, caso confirmado y contacto. El periodo de incubación es variable, pero generalmente dura de 2 a 7 días, aunque a veces puede ser hasta de 2 semanas; esto sugiere un periodo de cuarentena ideal mínimo de 14 días para paciente sintomático y 10 días para paciente asintomático. Se han establecido que la transmisión comienza entre 1 y 2 días antes del inicio de los síntomas. La 
enfermedad parece afectar un poco más a hombres que a mujeres, la mayoría de los afectados tienen edades que varían entre 30 y 79 años en el $87 \%$ de los casos, y se ha observado una menor susceptibilidad a COVID-19 en los menores, con tasas de enfermedad del $1 \%$ en los niños y jóvenes menores de 19 años, a pesar de tener cargas virales altas cuando se infectan (Guan et al, 2020).

Las categorías de pacientes con Covid-19 se han determinado en Infección asintomática o presintomática, enfermedad leve, enfermedad moderada, enfermedad grave y enfermedad crítica. La radiografía de tórax (RxT) en escenarios de la pandemia Sars-Cov-2 es una modalidad de imagen presente en la mayoría de los establecimientos de salud y es el primer estudio de imágenes para identificar la patología pulmonar (Huang, Wang, y Li, 2020).

La radiografía de tórax es generalmente la primera prueba de imagen en los pacientes con sospecha o confirmación de Covid-19 por su utilidad, disponibilidad y bajo costo, aunque es menos sensible y específico que la tomografía computarizada (TC) (23). El estudio óptimo incluye las proyecciones posteroanterior (PA) y lateral en bipedestación (Manna et al, 2020).

La realización de una radiografía de tórax portátil en proyección anteroposterior (AP) ayuda a una menor propagación de la infección, ya que estos equipos pueden limpiarse con facilidad y localizarse en instalaciones designadas para pacientes con Covid-19, lo que reduce la necesidad de transportar pacientes potencialmente infectados dentro del hospital y disminuye el uso de equipos de protección personal (EPP). Es la prueba radiológica recomendada en primera instancia por el American College of Radiology (ACR). Además, es la única posible en los pacientes críticos o ingresados en la $\mathrm{UCl}$. Su interpretación a menudo está limitada por el menor grado de inspiración y por la magnificación de la silueta cardiomediastínica derivada de la proyección AP. Pero, a pesar de las limitaciones, permite valorar la colocación de catéteres y dispositivos, detectar posibles complicaciones como neumotórax, enfisema subcutáneo y neumomediastino, y de forma seriada monitorizar la evolución de la enfermedad (Martínez et al, 2021).

En pacientes con SDRA, el puntaje Rale tuvo una asociación independiente tanto con la severidad del SDRA, la respuesta al manejo conservador de fluidos y desenlaces clínicos. Estos hallazgos, sugieren que el puntaje Rale provee un método nuevo e innovador para sacar provecho de información que se recolecta rutinariamente en pacientes con SDRA para evaluar de forma no invasiva tanto la extensión del edema pulmonar como la severidad de SDRA (Amaró, Solenzal, González, y Orellana, 2020). 
La escala más aceptada para evaluar la evolución de las imágenes radiográficas es el Score Rale propuesta por Warren et al, para cuantificar el edema pulmonar y adaptada por Wong et al, para evaluar progresión cuantitativa de la infección por SARS-COV-2. La escala consiste en dividir los campos pulmonares en 4 partes iguales de tal forma que se le asigne $25 \%$ a cada división y en cada pulmón por separado, con este procedimiento se consiguen 8 partes iguales (4 en el campo pulmonar derecho y 4 en el izquierdo), a las cuales se asigna 1 punto a cada área y se consigue una puntuación total de 8 si todos los segmentos estuvieran afectados. El score se determina sumando las áreas con imágenes sospechosas.

La escala para estratificación de la severidad en relación a la $\mathrm{Rx}$ de tórax es una modicación de RALE score (Radiographic Assessment of Lung Edema), diseñado para estimar el grado de edema pulmonar en el SDRA. Esta escala modificada, va de 0 a 8 puntos según la extensión de la afectación pulmonar. La puntuación de 0 a 4 puntos se obtiene calculando la afectación de cada pulmón por separado y sumándolos normalmente (Ureña y Bártulos, 2020).

Para calcularlo se divide visualmente en cuatro áreas cada pulmón siendo la mitad el hilio pulmonar. Cada área supone el $25 \%$ del parénquima pulmonar. Por cada área afectada con opacidades en vidrio esmerilado o consolidaciones se asigna 1 punto. Según la puntuación total, se gradúa radiológicamente la afectación pulmonar en: Normal: 0, leve: 1- 2, moderada: 3-6 y Severa: mayor a 6 puntos.

Las técnicas de imagen tienen un papel importante para apoyar el diagnóstico, graduar la gravedad de la enfermedad, guiar el tratamiento, detectar posibles complicaciones y valorar la respuesta terapéutica. La afectación del Covid-19, es principalmente pulmonar.

Los hallazgos radiológicos más frecuentes son las opacidades del espacio aéreo en forma de consolidaciones y/u opacidades en vidrio deslustrado, con distribución típicamente bilateral, periférica y de predominio en los campos inferiores.

Esta investigación es de importancia debido a la magnitud que ha tenido la pandemia del Covid-19, y a la diversidad de información relacionada con el diagnóstico de la enfermedad, el estudio pretende contribuir a dar un panorama real de otras investigaciones y la aplicación de la Score Rale, para identificar la afectación y la utilidad de las imágenes diagnósticas en infección respiratoria y apoyar la clasificación del riesgo y seguimiento de los pacientes con sospecha de neumonía pulmonar ocasionada por el Covid-19. 
En Guatemala en los hospitales destinados a pacientes de Covid-19, se realizan estudios de antígeno y PCR (reacción en cadena de la polimerasa), así como una evaluación radiológica si el paciente se encuentra con problemas respiratorios o ha ingresado con mayor gravedad.

La información recabada es de suma importancia para el personal médico y paramédico que realiza abordaje de pacientes con enfermedad de Covid-19, así como proporcionar mayor información sobre la importancia de los estudios radiológicos en el ámbito diagnóstico, podría utilizarse como predictor de la mortalidad de los pacientes con Covid- 19 detallando cuantitativa y cualitativamente el grado de afectación de edema pulmonar en pacientes diagnosticados con esta enfermedad.

\section{Conclusiones}

En el contexto de enfermedad de Covid-19, la radiografía de tórax puede ser normal en los casos leves o en las fases precoces de la enfermedad, sin embargo, en pacientes con afección pulmonar los hallazgos más frecuentes son las opacidades del espacio aéreo, ya sean las consolidaciones o, con menos frecuencia, las opacidades en vidrio deslustrado. Los hallazgos de la radiografía de tórax en pacientes con sospecha de Covid-19 se dividen en cuatro categorías: Radiografía de tórax normal, hallazgos típicos, hallazgos indeterminados y hallazgos atípicos.

Las complicaciones más comunes de la Covid-19 son la neumonía, el síndrome de dificultad respiratoria del adulto (SDRA), la miocarditis, el daño renal agudo y las sobreinfecciones bacterianas, frecuentemente en la forma de choque séptico, trastornos de la coagulación y compromiso de múltiples órganos.

La puntuación de Score Rale es un sistema cuantitativo de puntaje basado en la evaluación de la consolidación y la densidad de las opacidades alveolares en la radiografía de tórax, proporciona una valoración clínica significativa de la extensión del edema pulmonar en pacientes con SDRA, se utiliza para evaluar progresión cuantitativa de la infección por SARS-COV-2.

\section{Declaración}

El estudio se realizó con fines médicos, de acuerdo al Código de Ética y Buenas Prácticas COPE. 


\section{Conflicto de intereses}

El autor declara no tener ningún conflicto de intereses.

\section{Referencias}

Amaró, M., Solenzal, Y., González, T. y Orellana, G. (2020). Diagnóstico imagenológico de neumonía por SARS-CoV-2 en pacientes con la Covid-19. Gac Méd Espirit (en línea). 2020, vol.22, n.3, pp.175-193. http://scielo.sld.cu/scielo. php?script=sci_arttext\&pid=S1608-89212020000300175

American Veterinary Medical Association AVMA (2020). Coronavirus: detailed taxonomy Schaumburg, llinois, Estados Unidos. 2 p. https://www.avma.org/sites/ default/files/2020-02/AVMA-Detailed-Coronoavirus-Taxonomy-2020-02-03.pdf

Guan, W., Ni, Z., Hu, Y., Liang W., Ou, C., He J., Liu, L., y Shan, H. (2020). Clinical characteristics of coronavirus disease 2019 in China. The England Journal of Medicine 382:1708-1720. https://doi.org/10.1056/NEJMoa2002032 DOI: 10.1056/ NEJMoa2002032

Huang, C., Wang, Y., y Li, X. (2020). Clinical features of patients infected with 2019 novel coronavirus in Wuhan, China, Lancet, 2020; 395: 497-506. https://doi. org/10.1016/S0140-6736(20)30183-5

Manna, S., Wruble, J., Maron, S., Toussie, D., Voutsinas, N., Finkelstein, M., Cedillo, MA., Damond, J., Eber, C., Jacobi, A., Chung, M., Bernheim, A. (2020). COVID-19: a multimodality review of radiologic techniques. clinical utility, and imaging features. Radiology Cardiothoracic Imaging 2(3):e200210. https://doi. org/10.1148/ryct.2020200210

Martínez, E., Díez, T., Ibáñez, L., Ossaba, S., y Borruel, S. (2021). Diagnóstico radiológico del paciente con COVID-19. Radiología 63(1):56-73. https://doi.org/10.1016/j.rx.2020.11.001 
OPS (Organización Panamericana de la Salud); OMS (Organización Mundial de la Salud). (2021). Actualización epidemiológica: enfermedad por coronavirus (COVID-19). Washington, D.C., OPS/OMS. https://iris.paho.org/ handle/10665.2/53378

Ureña, A. y Bártulos, A. (2020). Estratificación severidad radiológica COVID 19, mediante Rx. https://doi.org/10.1148/radiol.2020201160

Wong, H., Lam, H., Fong, A., Leung S., Chin, T., Lo, C., Lui, M., Lee, J., Chiu, K., Wan, E. (2020). Frequency and distribution of chest radiographic findings in patients positive for COVID-19. Revista Radiology 296(2). https://pubs.rsna.org/ doi/10.1148/radiol.2020201160

\section{Sobre el autor \\ Roberto Carlos Barrientos Ramírez}

Estudiante de la carrera de médico y cirujano autor de la investigación "Trauma Craneoencefálico y uso de casco en pacientes con accidente en motocicletas en el Hospital de Chiquimula en el año 2019". Carrera de Médico y Cirujano, Centro Universitario de Oriente CUNORI, Facultad de Ciencias Médicas, Universidad de San Carlos de Guatemala.

Copyright (c) Roberto Carlos Barrientos Ramírez

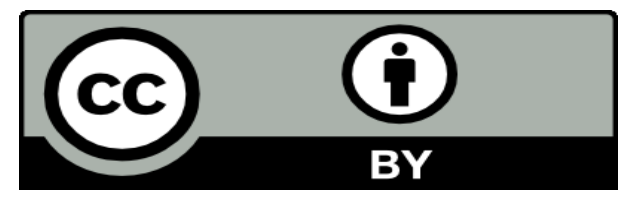

Este texto está protegido por una licencia CreativeCommons 4.0.

Usted es libre para compartir, copiar y redistribuir el material en cualquier medio o formato y adaptar el documento, remezclar, transformar y crear a partir del material para cualquier propósito, incluso comercialmente, siempre que cumpla la condición de atribución: usted debe reconocer el crédito de una obra de manera adecuada, proporcionar un enlace a la licencia, e indicar si se han realizado cambios. Puede hacerlo en cualquier forma razonable, pero no de forma tal que sugiera que tiene el apoyo del licenciante o lo recibe por el uso que hace. 